\title{
Neuroinflammation, Microglia and Implications for Anti-Inflammatory Treatment in Alzheimer's Disease
}

\author{
Daniela L. Krause and Norbert Müller \\ Department of Psychiatry and Psychotherapy, Ludwig-Maximilians University Munich, Nußbaumstr. 7, 80336 Munich, Germany \\ Correspondence should be addressed to Daniela L. Krause, daniela.krause@med.uni-muenchen.de
}

Received 15 February 2010; Accepted 28 April 2010

Academic Editor: Talma Brenner

Copyright ( 2010 D. L. Krause and N. Müller. This is an open access article distributed under the Creative Commons Attribution License, which permits unrestricted use, distribution, and reproduction in any medium, provided the original work is properly cited.

\begin{abstract}
Neuroinflammation has been implicated in the pathology of Alzheimer's disease (AD) for decades. Still it has not been fully understood when and how inflammation arises in the course of AD. Whether inflammation is an underling cause or a resulting condition in $\mathrm{AD}$ remains unresolved. Mounting evidence indicates that microglial activation contributes to neuronal damage in neurodegenerative diseases. However, also beneficial aspects of microglial activation have been identified. The purpose of this review is to highlight new insights into the detrimental and beneficial role of neuroinflammation in $\mathrm{AD}$. It is our intention to focus on newer controversies in the field of microglia activation. Precisely, we want to shed light on whether neuroinflammation is associated to brain tissue damage and functional impairment or is there also a damage limiting activity. In regard to this, we discuss the limitations and the advantages of anti-inflammatory treatment options and identify what future implications might result from this underling neuroinflammation for $\mathrm{AD}$ therapy.
\end{abstract}

\section{Introduction}

The pathology of Alzheimer's disease (AD) is characterized by the deposition of amyloid- $\beta(\mathrm{A} \beta)$ plaques in the brain parenchyma and neurofibrillary tangles within neurons [1].

Apart from the disease's distinct pathological markers, its neurodegenerative conditions are characterized by chronic neuroinflammatory processes. Yet, those inflammatory markers are not exclusively associated with $\mathrm{AD}$. Also brains of "healthy aged" individuals show concentrations of serum markers related to inflammation, homocysteine and cholesterol homeostasis are associated with cognitive functioning in the nondemented healthy aging population [2]. In the $\mathrm{AD}$ pathology, these aging-related inflammatory processes are increased. The suggestion that inflammation may participate in $\mathrm{AD}$ first came up more than two decades ago. As several clinical trials have shown a beneficial effect for nonsteroidal anti-inflammatory drugs for the occurrence and course of $\mathrm{AD}$, the inflammatory hypothesis in $\mathrm{AD}$ gained a lot of attention. In regard to treatment and prevention of $\mathrm{AD}$, several classes of medications have emerged to the market, which improve the cognitive symptoms of this disorder (e.g. the cholinesterase inhibitors). But the relief that these drugs provide remains symptomatic-so it is a major goal for the future to develop effective diseasemodifying therapy. Different substantial efforts have been made to identify potential strategies to ameliorate or prevent $\mathrm{AD}$ pathology, with data stemming from basic research as well as from animal and epidemiological studies. Because many investigators have concluded that neuroinflammation contributes to neuronal damage in the brain during $A D$ $[3,4]$, the use of anti-inflammatory drugs as a possible treatment option has been widely investigated [5-7]. Antiinflammatory therapy has therefore been credited as a strategy for reducing the risk or slowing the progression of AD. However, the results of these studies remain inconsistent [8].

Until now, many questions regarding the inflammatory response are still unresolved. Discussion continues whether neuroinflammation is an underling cause or a resulting condition in $\mathrm{AD}$.

There are several studies showing that an intact immune response including intact $\mathrm{T}$ cell immunity is a prerequisite for cognitive function. $\mathrm{T}$ cell deficient mice show impaired 
learning abilities, which can be reversed with $\mathrm{T}$ cell substitution $[9,10]$.

Inflammation in the brain is characterized by activation of glial cells (mainly microglia and astrocytes) and expression of key inflammatory mediators as well as neurotoxic free radicals. It has been suggested that neuroinflammation is associated with neurodegenerative disorders-both acute (e.g. stroke, injury) and chronic (e.g. multiple sclerosis, $\mathrm{AD})$. In this context, microglia cells play a crucial role and therefore microglia and cytokines have been extensively studied in these conditions. In the central nervous system, microglia are the resident phagocytes of the innate immune system. Microglia are found in a highly activated state in close anatomical proximity to senile plaques within the $\mathrm{AD}$ brain. In this activated state, microglia produce various proinflammatory cytokines and other immune mediators that create a neurotoxic milieu leading to disease progression $[4,11]$.

The purpose of this review is to highlight our new insights into the role of neuroinflammation in the pathophysiology of $\mathrm{AD}$. It is our intention to focus on newer controversies in the field of microglia activation and its function in $\mathrm{AD}$ pathology. For this, we asked ourselves some questions: are neuroinflammatory alterations neuroprotectiveor are they rather an underlying cause of $\mathrm{AD}$ ? And what strategies result from this underling neuroinflammation for future treatment options?

\section{Characteristics of Neuroinflammation in AD}

The relevance of neuroinammation to AD pathology has been established by multiple lines of direct and indirect evidence. One argument is that increased microglial activation has been shown in regions associated with $A \beta$ deposition [12]. Upregulated inammatory mechanisms colocalize in the $\mathrm{AD}$ brain with those regions that exhibit high levels of $\mathrm{AD}$ pathology (e.g. frontal and limbic cortex) and are minimal in brain regions with low $\mathrm{AD}$ pathologic susceptibility (e.g. cerebellum) [13].

As a second point, many of the inammatory mechanisms that have been uncovered in the $\mathrm{AD}$ brain are established to be cytotoxic in the periphery. Therefore it seems likely that they are also cytotoxic in the brain, an organ that is sensitive to inammation (e.g. meningitis, edema). However, inflammation in the brain is different from inflammation in the periphery. $\mathrm{AD}$ brains lack the classical hallmarks of inflammation such as neutrophil infiltration and perivascular mononuclear cuffing. As for other neurodegenerative diseases, a local inflammatory reaction is sustained by activated microglia and reactive astrocytes. This is indicated by the presence of antigens associated with microglia activation and inflammatory mediators, such as factors of the complement system, cytokines, and free radicals [14].

For $\mathrm{AD}$ a huge variety of proinflammatory markers have been identified, whereas this was not the case for other forms of dementia. A relevant reduction of monocyte chemotactic protein-1 levels in the grey matter in dementia patients has been shown. For interleukin-6 (IL) and related markers of this proinflammatory cytokine system, decreases were observed in the demented population $[15,16]$. It is discussed, however, whether this decrease is related to further psychopathological symptoms such as depression [16]. On the other hand, IL-6 has also neuroprotective properties and decreased IL- 6 might be associated with decreased neuroprotection [17],

Only modest elevations of inammatory markers are found in the autopsy of patients lacking a clinical presentation of dementia but who exhibit sufficient $A \beta$ and neurobrillary tangles to otherwise qualify for the diagnosis of AD. Their level of inflammatory markers is signicantly greater than levels of nondemented patients, but dramatically less than $\mathrm{AD}$ patients [18]. These findings further strengthen that an inflammation is a necessity for clinical symptoms of AD.

There also is direct evidence of inammatory toxicity in the $\mathrm{AD}$ brain. For instance, complement xation and lysis of neurites could be demonstrated ultrastructurally in Alzheimer's disease cortex, but in contrast it was only very weakly detected in nondemented elderly cortex under the same conditions [19].

Finally, many clinical and animal studies have strongly suggested that especially nonsteroidal anti-inflammatory drugs (NSAIDs) could be used as preventive or treatment strategies in $\mathrm{AD}$. This aspect is further discussed in a later section of this paper, where we focus on anti-inflammatory treatment.

Even though there are many indicators that neuroinflammation plays a key role in $\mathrm{AD}$ pathology, this does not answer which of these inflammatory activities are causing disease progression. The question remains: do some of these processes help to fight against the disease? In order to address this, the role of microglia seems important, because these cells are known for neuroprotective and degenerative functions.

\section{Controversy: Do Activated Microglia Cause Neuroprotection or -Degeneration in the $\mathrm{AD}$ Brain?}

Microglia are one of three glial cell types in the central nervous system (CNS). They play an important role as resident immunocompetent and phagocytic cells in the event of injury and disease. Del Rio Hortega determined in 1927 that microglia belong to a distinct glial cell type apart from astrocytes and oligodendrocytes [20]. Since the 1970s there has been wide recognition that microglia are immune effectors in the CNS that respond to pathological conditions and participate in initiation and progression of neurological disorders (including $\mathrm{AD}$ ) by releasing potentially cytotoxic molecules such as proinflammatory cytokines, reactive oxygen intermediates, proteinases, and complement proteins [21]. This means that their phagocytic function can be beneficial while their inflammation related functions might be detrimental.

Several studies give evidence for an increased number of morphologically reactive microglia in $\mathrm{AD}$ brains compared to nondemented individuals $[22,23]$. The location of these 
reactive microglia has been indentified directly around plaques [24]. This finding has been verified in a recent imaging study, which showed increased microglial activation in regions associated with amyloid deposition [12]. Up to now, the exact timing of this association could not be identified. Microgliosis might be an early component of the disease process and not necessarily dependent upon $\mathrm{A} \beta$ plaque interaction as a stimulus. What is known so far is that activation of microglia by $\mathrm{A} \beta$ fibrils is associated with a chemotactic response and extensive clustering of microglia around $\mathrm{A} \beta$ plaques in the $\mathrm{AD}$ brain [25]. These findings indicate the prominent role of microglial cells in $\mathrm{AD}$. Nonetheless it remains unclear, whether their functions are beneficial or detrimental.

The following section explains the checkered role of activated microglia in $\mathrm{AD}$ pathology.

\section{Neuroprotective Properties of Microglia in AD}

Is there a possibility that activated microglia cells are beneficial in neurodegenerative diseases? It is known that the microglia population can be neuroprotective by degrading $\mathrm{A} \beta$ plaques in $\mathrm{AD}$. Mouse models found that microglia mainly recruit macrophages from the periphery that then transform into microglia in the brain. Therefore most of the microglia that were associated with plaques in the mouse brain came from the bone marrow [26]. Furthermore it has been suggested that newly recruited microglia have different phagocytotic properties than intrinsic microglia, which is important for $A \beta$ elimination. Lysosmes from the macrophage cell line are more acidic than those of microglial lysosomes [27]. This indicates that microglia derived from the periphery might be more efficient in eliminating $A \beta$ than brain microglia. Furthermore, phagocytic activity of microglia is dampened by proinflammatory cytokines like tumor necrosis factor $\alpha$ (TNF) [28]. These findings show that microglia that are committed to an inflammatory response may have a lower phagocytotic capacity, than newly recruited microglia. In mouse models of $\mathrm{AD}$ it could be demonstrated that anti-inflammatory drugs like minocycline improve cognitive functions and reduce the activation of microglial cells but do not alter the $A \beta$ plaques deposition and distribution [29]. Seabrook et al. showed in amyloid precursor protein transgenic mice an age dependent effect of minocycline: in young animals the drug increased the amyloid load indicating a beneficial effect of microglia in clearing amyloid [30]. Not only for AD minocycline was investigated as a potential treatment, also in schizophrenia an add-on therapy with minocycline appeared to be effective on the cognitive performance by reducing a broad range of psychotic symptoms [31]. On the other hand an additional mechanism might help microglia cells with the elimination process. Transforming growth factor- $\beta 1$ has been demonstrated to promote microglial $\mathrm{A} \beta$ clearance and reduce plaque burden [32]. This could support the idea that microglial activation is useful in the clearance of $\mathrm{A} \beta$.

A further suggestion for the beneficial role of microglia is that neuroprotection results from the microglial glutamate removal. Glutamate has been indentified as a relevant neurotoxic substance that acts through $\mathrm{N}$-methyl-D-aspartic acid (NMDA) receptors on neurons and can lead to increased neuronal cell death. Microglial cells can increase their capacity to take up glutamate upon stimulation with lipopolysaccaride (LPS) over a mechanism that is TNF $\alpha$ dependent [33]. For $\mathrm{AD}$ this microglial function could be relevant because memantine (the NMDA receptor antagonist) has been shown to improve cognition, function (activities of daily living), agitation, and delusions in $\mathrm{AD}$ patients [34]. Taken this together, microglial cells are important for the control of glutamate levels and might therefore contribute to neuronal survival. There is also evidence that microglia are capable of secreting neurotrophic or neuron survival factors (e.g. nerve growth factor and neurotrophin 3) upon activation via inflammation or injury [35].

A recent review explains that microglia-when they are challenged-may adapt to different stimulatory contexts and pass through a sequence of reactive profiles. This is in line with the finding that microglia are not just "resting" but have active sensor and versatile functions [36].

Are most microglial cells functions beneficial in $\mathrm{AD}$ ? Several studies suggest an overbalance of the detrimental microglial properties. This issue is discussed in the next section.

\section{Microglia-Are They Responsible for Neurodestruction and -Degeneration?}

In order to address this question, it is important to focus on timing. One must investigate when microglial activity begins during the time course of the disease. An increase in microglial activation has been observed in very early stages of AD. This increase surprisingly disappeared over time [37]. The suggestion of Vehmas et al. strengthens the assumption that microglial activation begins early in disease progression [37]. This could be a hint that microglia initially try to eliminate $\mathrm{A} \beta$, but over time of the disease fail and therefore decrease their activity. Alternatively, the microglial role in $\mathrm{AD}$ could be detrimental and they initiate the underlying $\mathrm{AD}$ pathology. In order to further evaluate this issue, a closer look needs to be taken on what causes the microglial activation in $\mathrm{AD}$ and it seems important to distinguish between acute and chronic stimulation of microglial cells. While an acute insult may trigger oxidative and nitrosative stress, it is typically short-lived and unlikely to be harmful to long-term neuronal survival. Therefore it is believed that an acute neuroinflammatory response is generally beneficial to the CNS, since it tends to minimize further injury and contributes to repair of damaged tissue. The opposite is the case for a chronic stimulation: chronic neuroinflammation is most often detrimental and damaging to nervous tissue. Thus, whether neuroinflammation has beneficial or harmful outcomes in the brain may depend critically on the duration of the inflammatory response. The progressive deposition of $\mathrm{A} \beta$ in $\mathrm{AD}$ disease might provide a chronic stimulus to microglial cells. Also the chemotactic functions of $A \beta$ to attract microglia contribute further to the ongoing 
inflammatory process [25]. The ratio of the proinflammatory cytokine IL-1 $\beta$ to the anti-inflammatory cytokine IL-10 is drastically elevated in the serum of $\mathrm{AD}$ patients, giving these patients a definite long-term proinflammatory profile [38], indicating a chronic neuroinflammatory state of the CNS. In addition, the accumulating loss of neurons that characterizes $\mathrm{AD}$ further contributes to generation of debris and keeps microglia activated indefinitely maintaining microglia in an activated state long term. This data indicates that in $\mathrm{AD}$ the inflammation might be rather chronic and therefore contributing to disease progression.

There is also the emerging idea that an inflamed CNS environment may influence the ability of microglia to contribute to plaque deposition rather than plaque removal [28]. This strongly suggests that the microenvironment of the brain can influence whether microglia perform beneficial or deleterious functions in pathophysiological states. This means that microglia cells functionally adapt to their environment [36]. Recent studies show that in response to certain environmental toxins and endogenous proteins, microglia can enter an overactivated state and release reactive oxygen species (ROS) that cause neurotoxicity [39]. Overactivated microglia can be detected using imaging techniques and therefore this knowledge offers an opportunity not only for early diagnosis, but eventually also for the development of targeted anti-inflammatory therapies that might diminish the progression of the disease [21].

In addition, activated microglia release the excitotoxin quinolinic acid [40], and microglia activated by $\mathrm{AD}$ plaques produce an apparently novel amine that evokes fulminant excitotoxicity [41]. One interesting implication of an excitotoxic contribution to inammatory mechanisms is the potential for limited damage to functional cellular compartments. Because excitatory amino acid receptors are restricted to synapses and dendrites, these subcellular compartments are preferentially vulnerable.

As a result, microglia-produced excitotoxins may lead to cognitive impairment that is not necessarily correlated with neuronal cell loss [3]. However, activated microglia do not only produce neurotoxic metabolites. Some of their products like 3-hydroxyanthralinic acid (which islike quinolinic acid-one of the downstream products of the tryptophan metabolism) exert antioxidant and antiinflammatory functions $[42,43]$. Therefore the balance of these products that result from activated microglia is important for the inflammation process.

To sum up the results from microglial studies: clear indications for the important role of neuroinflammation contributing to disease progression in $\mathrm{AD}$ were found. However, some parts of microglial activation might also be beneficial during the course of $\mathrm{AD}$. These issues are shown in Figure 1.

\section{The Role of COX Inhibitors in Neurodegeneration}

As explained above, neuroinflammation is a critical event in $\mathrm{AD}$. It has been suggested that anti-inammatory therapy could be benecial in delaying the onset or slowing the progression of $\mathrm{AD}$.

Cyclooxygenase (COX) is a unique enzyme. First, it exhibits two catalytic activities, a bis-oxygenase activity, which catalyses prostaglandin $G_{2}$ (PG) formation from arachidonic acid and a peroxidase activity, which reduces $P G G_{2}$ to $P G H_{2}$. The peroxidase activity also results in the production of free radicals, which are in part utilized by COX itself [44]. Although NSAIDs may have other effects as well, it is generally assumed that their primary mechanism of action is by competitive inhibition of COX activity, thereby reducing the production of inammatory prostaglandins from membrane-derived arachidonate. COX not only helps mediate production of prostaglandins and other inammatory factors, it is itself upregulated by proinammatory mediators [44].

In $\mathrm{AD}, \mathrm{A} \beta$ neurotoxicity may result from several mechanisms, most likely in combination. These mechanisms include oxidative damage, direct cytotoxicity, and induction of destructive inflammatory mechanisms; efforts have been directed at the control of each of these processes [45]. See Figure 1 for the involvment of COX in the AD pathology.

The treatment of AD with NSAIDs is one of the most promising approaches.

\section{Possible Mechanisms of Action of NSAID in AD}

If NSAIDs are beneficial in $\mathrm{AD}$, the presumed mechanism would be inhibition of COX expressed in the brain. Both COX-1 and COX-2 are expressed there and COX-2 plays a unique role in the brain compared to the periphery: only in the brain COX-2 is expressed constitutively whereas elsewhere the expression is activation-dependent. Although in vivo the majority of COX-2 appears to be made in neurons, COX-2 was also seen in rat astrocytes and microglia [46]. It has been demonstrated that COXinhibiting NSAIDs reduce microglial activation following infusion of $A \beta$ in rats [47]. Neuronal stress, such as ischaemia and excitotoxicity, is associated with strong upregulation of neuronal COX-2 expression. This suggests that COX-2 is involved in neurotoxic mechanisms and may therefore represent a target for drug therapy in the treatment of $\mathrm{AD}$ $[48,49]$.

Several studies provide the background for possible mechanisms of action of NSAIDs in AD. Neuronal COX-2 is upregulated in response to exposure to $A \beta$ [50], and focal increases in COX-2 have been shown in the region of amyloid plaques in double transgenic mice carrying genes that encode both mutant APP and mutant presenilin 1 [51]. Many studies seem to show that COX-2 inhibition confers neuroprotection [52-55]. Some studies have revealed an upregulation of neuronal COX-2 in the brains of patients with $\mathrm{AD}[56,57]$, though this has not been a universal finding $[58,59]$. One explanation for the variation of COX expression is the short half-life of COX-2 transcripts or individual variability of inflammatory-related processes. 


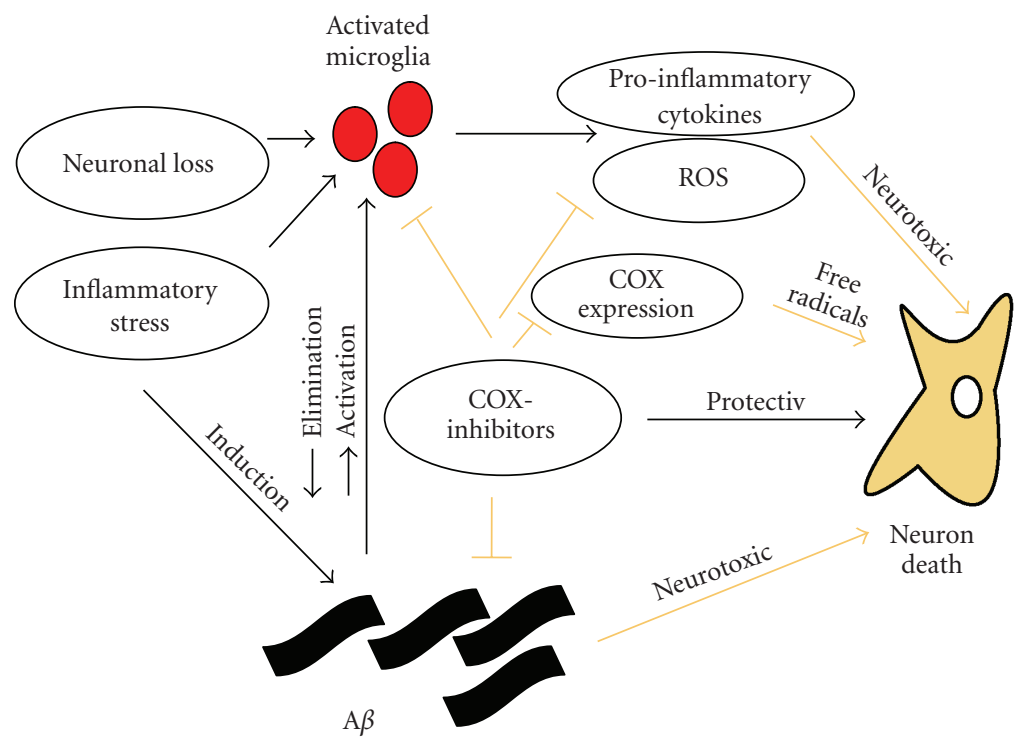

FIGURE 1: possible interactions of COX-inhibitors and Alzheimer's disease pathology. The fair arrows show neurotoxic properties of A $\beta$, COX-expression cytokines. In addition it is indicated that COX-inhibitors block COX-expression, activated microglia, ROS, and A $\beta$. ROS: radical oxygen species; COX: cyclooxygenase; $\mathrm{A} \beta$ : amyloid $\beta$.

Another principle of how NSAIDs could act, comes from the finding that prostaglandin E2 levels are elevated in patients with $\mathrm{AD}$, especially in early stages of the disease [60]. Therefore NSAIDs blocking prostaglandin E2 synthesis might be beneficial. This issue is further strengthened by glial culture studies indicating that prostaglandins, particularly prostaglandin E, alter the production of several inammation-related molecules, including IL-6, chemokines, and APP [61-63].

In addition to the more traditional inammatory mechanisms associated with COX, unique functions of COXmediated damage may also occur in the AD brain. For example, several of the prostanoid products of arachidonate metabolism potentiate glutamate excitotoxicity, and COX2 overexpressing transgenic mice exhibit increased neuronal susceptibility to excitotoxic insult [64].

Some of the previously mentioned studies of COX in ischemia also suggest that intraneuronal COX-2 levels may contribute to neuronal death by production of free radicals [65]. In addition, increased COX-2 levels in AD neurons may directly damage neurons or increase their vulnerability to other detrimental processes occurring in $\mathrm{AD}$ brain [65]. Thus, NSAIDs actions to inhibit COX-mediated production of apoptotic factors by neurons could be one of the mechanisms by which these drugs seem to exert benecial effects in AD.

Another non-COX-dependent mechanism of NSAIDs is to attenuate inammatory processes in a manner by directly activating the peroxisome proliferator-activated receptor gamma $(\operatorname{PPAR} \gamma)$, a receptor and nuclear transcription factor [66-68]. PPAR $\gamma$ is a member of the orphan nuclear receptor family and in cells of monocytic lineage, including microglia, acts to suppress the expression of a broad range of proinammatory genes $[66,68]$. Some NSAIDs act as
PPAR $\gamma$ agonists, directly binding to it and initiating its transcriptional activity. Activation of PPAR $\gamma$ inhibits the A $\beta$ stimulated activation of microglia and monocytes and their secretion of proinammatory and neurotoxic products. For example, PPAR $\gamma$ agonists act to inhibit the A $\beta$-stimulated expression of IL-6 and TNF-alpha [69], by microglia and monocytes, and to prevent $\mathrm{A} \beta$-mediated conversion of microglia into an activated phenotype [70].

A further underlying mechanism of $\mathrm{AD}$ pathology is oxidative stress $[71,72]$. Activated microglial cells are known to release ROS, which might possibly cause this oxidative stress. Though glia cells can also exhibit antioxidative functions by releasing hemeoxygenase-1 (HO-1) triggered by accumulation of 3-hydroxyanthralinic acid (3-HAA), a down-stream product of the tryptophan metabolism. The association of neuronal injury in $\mathrm{AD}$ and oxidative stress has been demonstrated by overexpression of immunoreactive HO-1 protein in neurons and astrocytes of the cerebral cortex and hippocampus. HO-1 was found to be colocalized to senile plaques, neurofibrillary tangles, and corpora amylacea [73]. It is widely accepted that a moderate activation of heme catabolism is neuroprotective and contributes to degradation of neurotoxic protein aggregates. Regulatory interactions between HO-1 and COX pathways have also been reported [74]. However, experimental observations indicate that the extent of HO-1 induction may be critical because excessive heme degradation may result in toxic levels of carbon monoxide, bilirubin and iron. Pharmacological modulation of HO-1 levels in the brain shows promising results in models of $\mathrm{AD}$ and Parkinson's disease [75].

Referring to the oxidative stress underlying AD pathology, one further aspect of these reactive oxygen species includes activation of COX-1/2, which are blocked by NSAIDs. It has been shown that daily doses of NSAIDs 
increase circulating levels of antioxidants [76]. In a rat model of $\mathrm{AD}$ it was suggested that treatment with a COX-2 inhibitor reduces oxidative stress and might therefore be beneficial for the course of AD [77].

As another mechanism it has been suggested that NSAIDs directly affect amyloid pathology in the brain by reducing $\mathrm{A} \beta-42$ peptide levels over the gamma-secretase activity independently of COX activity [78]. Weggen et al. reported that the NSAIDs ibuprofen, indomethacin, and sulindac sulphide preferentially decrease the highly amyloidogenic $\mathrm{A} \beta-42$ peptide produced from a variety of cultured cells by as much as $80 \%$ [79]. However, for some NSAIDs the lowering effect of $\mathrm{A} \beta-42$ could not be shown; instead, an increase in $\mathrm{A} \beta-42$ levels was observed [80]. The underlying mechanism of how NSAIDs decrease $A \beta-42$ was clarified by Lleo et al., who demonstrated that $\mathrm{A} \beta-42$ lowering NSAIDs specifically affect the proximity between APP and presenilin 1 and alter a novel allosteric mechanism of action [81].

\section{Anti-Inflammatory Treatment Studies in AD}

In recent years it has become widely accepted that inflammatory processes are an underlying condition of $\mathrm{AD}$. Therefore a number of clinical trials investigating different antiinflammatory treatment regimens have been performed. In the following paragraph, we summarize the most import findings in regard to first mainly COX-2 dominant and second COX-1 inhibitors.

A prospective cohort study with 6989 subjects showed that long-term use of NSAIDs protects against AD but not against vascular dementia [5]. More recently, Szekely et al. provided very similar findings: they concluded that NSAIDs use reduced the risk of preferentially AD versus vascular dementia but mainly in those individuals having an apolipoprotein E (APO) epsilon 4 allele. This study was done with over 3,000 subjects aged 65 years and older [6]. Not only selective COX-2 inhibitors were shown to be associated with decreased risk of $\mathrm{AD}$; a reduced occurrence of $\mathrm{AD}$ could also be demonstrated for the use of the COX-1 inhibitor aspirin [7]. A meta-analysis of 17 epidemiological studies yielded strong, generally consistent, statistical evidence that NSAID and steroid use is associated with reduced risk of $\mathrm{AD}$ [82]. Vlad et al. investigated 49,349 patients with AD and 196,850 controls: long-term (>5 years) nonsteroidal anti-inflammatory drug use was shown to be protective against Alzheimer disease. These findings were clearest for ibuprofen, but did not appear for other NSAIDs [83].

Not all studies showed a positive outcome for COX inhibitors in $\mathrm{AD}$ patients: the failure of selective COX-2 inhibition (rofecoxib) over placebo was stated in a oneyear randomized controlled study. The authors argued that their results could indicate that the disease process was too advanced to be modified, as the goal of the study was slowing the progression of dementia in patients with already established AD [8]. For another COX-2 inhibitor, celecoxib, no beneficial effect on the occurrence of $\mathrm{AD}$ could be demonstrated in an age group over 70 years [84]. Also Wolfson et al. looked retrospectively at a case control population and found no support for a beneficial effect for NSAIDs in the AD subjects [85]. However, this negative result may have been caused by an insufficient period of data collection before disease onset.

\section{Conclusion}

It is indisputable that neuroinammation plays a key role in AD pathology. Mechanisms that parallel those encountered in localized peripheral inammatory responses are readily identied, along with detailed pathways for how the mechanisms interact. On balance, it is likely that $\mathrm{AD}$ neuroinammation exacerbates $\mathrm{AD}$ pathogenesis.

A general treatment principle in psychiatry, that an intervention as early as possible leads to the best outcome, seems to be especially true for AD. Many lines of evidence show that $\mathrm{A} \beta$-induced neuroinflammation is an early event in neurodegeneration of $\mathrm{AD}$ [86], as increases in microglial activation has been observed in very early stages of $A D$ and disappeared over time [37]. The fact that neuroinflammation occurs very early in AD could explain why anti-inflammatory treatment seems to be most efficient as preventive or early treatment. There are several reasons why an early use of NSAIDs is superior to a late one: Cox-expression in the brain decreases over time in $\mathrm{AD}$ brains [87]. And the CSF PG $E_{2}$ levels in patients with Alzheimer's disease were high when their short-term memory scores were just below those of controls, but were low in later stages of the disease. These findings further support that inflammatory processes predominate early in Alzheimer's disease [88] and therefore require early intervention with anti-inflammatory treatment.

This could explain the failure of some prospective clinical trials of selective COX-2 inhibitors: it may be related to a delayed onset of treatment, but eventually also to drug selection (regarding different effects of COX-1 and COX2) and dose and duration of treatment. Especially the drug selection seems essential as some NSAIDs have recently been shown to increase $\mathrm{A} \beta-42$ levels [77]. It also has to be noted that the protective effects of NSAIDs may be via non-COXinhibitory mechanisms, such as lowering of $\mathrm{A} \beta$ levels and activation of the peroxisome proliferator-activated receptor[gamma] [89] and these non-COX-dependent mechanisms might be differentially distributed among COX-inhibitors.

However, two major aspects should be kept in mind when considering the significance of COX-2 activity in brain diseases. The first thing: COX-2 is expressed under normal conditions and contributes to fundamental brain functions such as synaptic activity, memory consolidation, and functional hyperemia. The second thing: the term neuroinflammation is a much more controlled reaction than inflammation in peripheral tissues. In degenerative diseases, it mainly occurs in the absence of blood-borne infiltrating cells and is sustained by activated glial cells, particularly microglia.

In summary, the harmful inflammatory processes seem to dominate $\mathrm{AD}$ pathology, but there are also some beneficial functions for inflammatory subsets. If AD neuroinammation is approached with realistic expectations and rational drug 
design, AD patients could significantly benefit from antiinammatory treatment, especially with NSAIDs.

A future goal could be to utilize not only the efficient treatment properties of NSAIDs in early AD, but also makes use of the neuroprotective aspects of neuroinflammation with a combination therapy that maximizes the potential of glial activation. This would include treatment with NSAIDs and drugs that enforce anti-inflammatory and antioxidative properties (e.g. with 3-HAA and HO-1 enhancement).

\section{References}

[1] J. Götz and N. N. Götz, "Animal models for Alzheimer's disease and frontotemporal dementia: a perspective," ASN Neuro, vol. 1, no. 4, article e00019, 2009.

[2] C. E. Teunissen, M. P. J. van Boxtel, H. Bosma, et al., "Serum markers in relation to cognitive functioning in an aging population: results of the Maastricht Aging Study (MAAS)," Tijdschrift voor Gerontologie en Geriatrie, vol. 34, no. 1, pp. 6 12, 2003.

[3] E. G. McGeer and P. L. McGeer, "Neuroinflammation in Alzheimer's disease and mild cognitive impairment: a field in its infancy," Journal of Alzheimer's Disease, vol. 19, no. 1, pp. 355-361, 2010.

[4] H. Akiyama, S. Barger, S. Barnum, et al., "Inflammation and Alzheimer's disease," Neurobiology of Aging, vol. 21, no. 3, pp. 383-421, 2000.

[5] B. A. in 't Veld, A. Ruitenberg, A. Hofman, et al., "Nonsteroidal antiinflammatory drugs and the risk of Alzheimer's disease," The New England Journal of Medicine, vol. 345, no. 21, pp. 1515-1521, 2001.

[6] C. A. Szekely, J. C. S. Breitner, A. L. Fitzpatrick, et al., "NSAID use and dementia risk in the Cardiovascular Health Study: role of APOE and NSAID type," Neurology, vol. 70, no. 1, pp. 1724, 2008.

[7] J. C. Anthony, J. C. S. Breitner, P. P. Zandi, et al., "Reduced prevalence of $\mathrm{AD}$ in users of NSAIDS and $\mathrm{H} 2$ receptor antagonists: the Cache County study," Neurology, vol. 54, no. 11, pp. 2066-2071, 2000.

[8] S. A. Reines, G. A. Block, J. C. Morris, et al., "No effect on Alzheimer's disease in a 1-year, randomized, blinded, controlled study," Neurology, vol. 62, no. 1, pp. 66-71, 2004.

[9] J. Kipnis, H. Cohen, M. Cardon, Y. Z IV, and M. Schwartz, "T cell deficiency leads to cognitive dysfunction: implications for therapeutic vaccination for schizophrenia and other psychiatric conditions," Proceedings of the National Academy of Sciences of the United States of America, vol. 101, no. 21, pp. 8180-8185, 2004.

[10] Y. Ziv, N. Ron, O. Butovsky, et al., "Immune cells contribute to the maintenance of neurogenesis and spatial learning abilities in adulthood," Nature Neuroscience, vol. 9, no. 2, pp. 268-275, 2006.

[11] T. Wyss-Coray, "Inflammation in Alzheimer disease: driving force, bystander or beneficial response?" Nature Medicine, vol. 12, no. 9, pp. 1005-1015, 2006.

[12] P. Edison, H. A. Archer, A. Gerhard, et al., "Microglia, amyloid, and cognition in Alzheimer's disease: an [11C](R)PK11195PET and [11C]PIB-PET study," Neurobiology of Disease, vol. 32, no. 3, pp. 412-419, 2008.

[13] J. Rogers and Y. Shen, "A perspective on inflammation in Alzheimer's disease," Annals of the New York Academy of Sciences, vol. 924, pp. 132-135, 2000.
[14] V. H. Perry, T. A. Newman, and C. Cunningham, "The impact of systemic infection on the progression of neurodegenerative disease," Nature Reviews Neuroscience, vol. 4, no. 2, pp. 103$112,2003$.

[15] E. Mulugeta, F. Molina-Holgado, M. S. Elliott, et al., "Inflammatory mediators in the frontal lobe of patients with mixed and vascular dementia," Dementia and Geriatric Cognitive Disorders, vol. 25, no. 3, pp. 278-286, 2008.

[16] S. Stübner, T. Schön, F. Padberg, et al., "Interleukin-6 and the soluble IL- 6 receptor are decreased in cerebrospinal fluid of geriatric patients with major depression: no alteration of soluble gp130," Neuroscience Letters, vol. 259, no. 3, pp. 145$148,1999$.

[17] X.-Q. Wang, Y.-P. Peng, J.-H. Lu, B.-B. Cao, and Y.-H. Qiu, "Neuroprotection of interleukin-6 against NMDA attack and its signal transduction by JAK and MAPK," Neuroscience Letters, vol. 450, no. 2, pp. 122-126, 2009.

[18] L.-F. Lue, L. Brachova, W. H. Civin, and J. Rogers, "Inflammation, $\mathrm{A} \beta$ deposition, and neurofibrillary tangle formation as correlates of Alzheimer's disease neurodegeneration," Journal of Neuropathology and Experimental Neurology, vol. 55, no. 10, pp. 1083-1088, 1996.

[19] S. Webster, L.-F. Lue, L. Brachova, et al., "Molecular and cellular characterization of the membrane attack complex, C5b-9, in Alzheimer's disease," Neurobiology of Aging, vol. 18, no. 4, pp. 415-421, 1997.

[20] P. del Rio-Hortega, "Art and artifice in the science of histology," Histopathology, vol. 22, no. 6, pp. 515-525, 1993.

[21] M. L. Block, L. Zecca, and J.-S. Hong, "Microglia-mediated neurotoxicity: uncovering the molecular mechanisms," Nature Reviews Neuroscience, vol. 8, no. 1, pp. 57-69, 2007.

[22] P. Cras, M. Kawai, S. Siedlak, et al., "Neuronal and microglial involvement in $\beta$-amyloid protein deposition in Alzheimer's disease," American Journal of Pathology, vol. 137, no. 2, pp. 241-246, 1990.

[23] S. D. Styren, W. H. Civin, and J. Rogers, "Molecular, cellular, and pathologic characterization of HLA-DR immunoreactivity in normal elderly and Alzheimer's disease brain," Experimental Neurology, vol. 110, no. 1, pp. 93-104, 1990.

[24] L. S. Perlmutter, E. Barron, and H. C. Chui, "Morphologic association between microglia and senile plaque amyloid in Alzheimer's disease," Neuroscience Letters, vol. 119, no. 1, pp. 32-36, 1990.

[25] L.-F. Lue, R. Rydel, E. F. Brigham, et al., "Inflammatory repertoire of Alzheimer's disease and nondemented elderly microglia in vitro," GLIA, vol. 35, no. 1, pp. 72-79, 2001.

[26] A. R. Simard and S. Rivest, "Bone marrow stem cells have the ability to populate the entire central nervous system into fully differentiated parenchymal microglia," FASEB Journal, vol. 18, no. 9, pp. 998-1000, 2004.

[27] A. Majumdar, D. Cruz, N. Asamoah, et al., "Activation of microglia acidifies lysosomes and leads to degradation of Alzheimer amyloid fibrils," Molecular Biology of the Cell, vol. 18, no. 4, pp. 1490-1496, 2007.

[28] J. Koenigsknecht-Talboo and G. E. Landreth, "Microglial phagocytosis induced by fibrillar $\beta$-amyloid and IgGs are differentially regulated by proinflammatory cytokines," Journal of Neuroscience, vol. 25, no. 36, pp. 8240-8249, 2005.

[29] R. Fan, F. Xu, M. L. Previti, et al., "Minocycline reduces microglial activation and improves behavioral deficits in a transgenic model of cerebral microvascular amyloid," Journal of Neuroscience, vol. 27, no. 12, pp. 3057-3063, 2007. 
[30] T. J. Seabrook, L. Jiang, M. Maier, and C. A. Lemere, "Minocycline affects microglia activation, $\mathrm{A} \beta$ deposition, and behavior in APP-tg mice," GLIA, vol. 53, no. 7, pp. 776-782, 2006.

[31] C. Chaves, C. R. Marque, C. Trzesniak, et al., "Glutamate$\mathrm{N}$-methyl-D-aspartate receptor modulation and minocycline for the treatment of patients with schizophrenia: an update," Brazilian Journal of Medical and Biological Research, vol. 42, no. 11, pp. 1002-1014, 2009.

[32] T. Wyss-Coray, C. Lin, F. Yan, et al., "TGF- $\beta 1$ promotes microglial amyloid- $\beta$ clearance and reduces plaque burden in transgenic mice," Nature Medicine, vol. 7, no. 5, pp. 612-618, 2001.

[33] M. Persson, M. Brantefjord, E. Hansson, and L. Rönnbäck, "Lipopolysaccharide increases microglial GLT-1 expression and glutamate uptake capacity in vitro by a mechanism dependent on TNF- $\alpha$," GLIA, vol. 51, no. 2, pp. 111-120, 2005.

[34] P. T. Francis, "Altered glutamate neurotransmission and behaviour in dementia: evidence from studies of memantine," Current Molecular Pharmacology, vol. 2, no. 1, pp. 77-82, 2009.

[35] S. U. Kim and J. de Vellis, "Microglia in health and disease," Journal of Neuroscience Research, vol. 81, no. 3, pp. 302-313, 2005.

[36] U.-K. Hanisch and H. Kettenmann, "Microglia: active sensor and versatile effector cells in the normal and pathologic brain," Nature Neuroscience, vol. 10, no. 11, pp. 1387-1394, 2007.

[37] A. K. Vehmas, C. H. Kawas, W. F. Stewart, and J. C. Troncoso, "Immune reactive cells in senile plaques and cognitive decline in Alzheimer's disease," Neurobiology of Aging, vol. 24, no. 2, pp. 321-331, 2003.

[38] E. J. Remarque, E. L. E. M. Bollen, A. W. E. WeverlingRijnsburger, J. C. Laterveer, G. J. Blauw, and R. G. J. Westendorp, "Patients with Alzheimer's disease display a proinflammatory phenotype," Experimental Gerontology, vol. 36, no. 1, pp. 171-176, 2001.

[39] N. G. Innamorato, I. Lastres-Becker, and A. Cuadrado, "Role of microglial redox balance in modulation of neuroinflammation," Current Opinion in Neurology, vol. 22, no. 3, pp. 308314, 2009.

[40] M. G. Espey, O. N. Chernyshev, J. F. Reinhard Jr., M. A. A. Namboodiri, and C. A. Colton, "Activated human microglia produce the excitotoxin quinolinic acid," NeuroReport, vol. 8, no. 2, pp. 431-434, 1997.

[41] D. Giulian, "Senile plaques stimulate microglia to release a neurotoxin found in Alzheimer brain," Neurochemistry International, vol. 27, no. 1, pp. 119-137, 1995.

[42] G. Leipnitz, C. Schumacher, K. B. Dalcin, et al., "In vitro evidence for an antioxidant role of 3-hydroxykynurenine and 3-hydroxyanthranilic acid in the brain," Neurochemistry International, vol. 50, no. 1, pp. 83-94, 2007.

[43] S. R. Thomas, P. K. Witting, and R. Stocker, "3hydroxyanthranilic acid is an efficient, cell-derived coantioxidant for $\alpha$-tocopherol, inhibiting human low density lipoprotein and plasma lipid peroxidation," Journal of Biological Chemistry, vol. 271, no. 51, pp. 32714-32721, 1996.

[44] W. L. Smith, R. Michael Garavito, and D. L. DeWitt, "Prostaglandin endoperoxide $\mathrm{H}$ syntheses (cyclooxygenases)1 and -2," Journal of Biological Chemistry, vol. 271, no. 52, pp. 33157-33160, 1996.

[45] P. S. Aisen and K. L. Davis, "The search for disease-modifying treatment for Alzheimer's disease," Neurology, vol. 48, no. 5, pp. S35-S41, 1997.
[46] W. D. Hirst, K. A. Young, R. Newton, V. C. Allport, D. R. Marriott, and G. P. Wilkin, "Expression of COX-2 by normal and reactive astrocytes in the adult rat central nervous system," Molecular and Cellular Neurosciences, vol. 13, no. 1, pp. 57-68, 1999.

[47] B. Hauss-Wegrzyniak, P. Vraniak, and G. L. Wenk, "The effects of a novel NSAID on chronic neuroinflammation are age dependent," Neurobiology of Aging, vol. 20, no. 3, pp. 305-313, 1999.

[48] A. M. Planas, M. A. Soriano, E. Rodríquez-Farré, and I. Ferrer, "Induction of cyclooxygenase-2 mRNA and protein following transient focal ischemia in the rat brain," Neuroscience Letters, vol. 200, no. 3, pp. 187-190, 1995.

[49] G. Tocco, J. Freire-Moar, S. S. Schreiber, S. H. Sakhi, P. S. Aisen, and G. M. Pasinetti, "Maturational regulation and regional induction of cyclooxygenase-2 in rat brain: implications for Alzheimer's disease," Experimental Neurology, vol. 144, no. 2, pp. 339-349, 1997.

[50] G. M. Pasinetti and P. S. Aisen, "Cyclooxygenase-2 expression is increased in frontal cortex of Alzheimer's disease brain," Neuroscience, vol. 87, no. 2, pp. 319-324, 1998.

[51] Y. Matsuoka, M. Picciano, B. Maleste, et al., "Inflammatory responses to amyloidosis in a transgenic mouse model of Alzheimer's disease," American Journal of Pathology, vol. 158, no. 4, pp. 1345-1354, 2001.

[52] S. J. Hewett, T. F. Uliasz, A. S. Vidwans, and J. A. Hewett, "Cyclooxygenase-2 contributes to N-methyl-D-aspartatemediated neuronal cell death in primary cortical cell culture," Journal of Pharmacology and Experimental Therapeutics, vol. 293, no. 2, pp. 417-425, 2000.

[53] L. B. Willard, B. Hauss-Wegrzyniak, W. Danysz, and G. L. Wenk, "The cytotoxicity of chronic neuroinflammation upon basal forebrain cholinergic neurons of rats can be attenuated by glutamatergic antagonism or cyclooxygenase-2 inhibition," Experimental Brain Research, vol. 134, no. 1, pp. 58-65, 2000.

[54] T. Kunz and E. H. Oliw, "The selective cyclooxygenase-2 inhibitor rofecoxib reduces kainate-induced cell death in the rat hippocampus," European Journal of Neuroscience, vol. 13, no. 3, pp. 569-575, 2001.

[55] E. Araki, C. Forster, J. M. Dubinsky, M. E. Ross, and C. Iadecola, "Cyclooxygenase-2 inhibitor NS-398 protects neuronal cultures from lipopolysaccharide-induced neurotoxicity," Stroke, vol. 32, no. 10, pp. 2370-2375, 2001.

[56] K. Yasojima, C. Schwab, E. G. McGeer, and P. L. McGeer, "Distribution of cyclooxygenase- 1 and cyclooxygenase-2 mRNAs and proteins in human brain and peripheral organs," Brain Research, vol. 830, no. 2, pp. 226-236, 1999.

[57] L. Ho, D. Purohit, V. Haroutunian, et al., "Neuronal cyclooxygenase 2 expression in the hippocampal formation as a function of the clinical progression of Alzheimer disease," Archives of Neurology, vol. 58, no. 3, pp. 487-492, 2001.

[58] W. J. Lukiw and N. G. Bazan, "Cyclooxygenase 2 RNA message abundance, stability, and hypervariability in sporadic Alzheimer neocortex," Journal of Neuroscience Research, vol. 50, no. 6, pp. 937-945, 1997.

[59] J. W. Chang, P. D. Coleman, and M. K. O'Banion, "Prostaglandin G/H synthase-2 (cyclooxygenase-2) mRNA expression is decreased in Alzheimer's disease," Neurobiology of Aging, vol. 17, no. 5, pp. 801-808, 1996.

[60] T. J. Montine, K. R. Sidell, B. C. Crews, et al., "Elevated CSF prostaglandin E2 levels in patients with probable AD," Neurology, vol. 53, no. 7, pp. 1495-1498, 1999. 
[61] R. K. K. Lee, S. Knapp, and R. J. Wurtman, "Prostaglandin E2 stimulates amyloid precursor protein gene expression: inhibition by immunosuppressants," Journal of Neuroscience, vol. 19, no. 3, pp. 940-947, 1999.

[62] M. A. A. Blom, M. G. H. Van Twillert, S. C. de Vries, et al., "NSAIDS inhibit the IL- $1 \beta$-induced IL-6 release from human post-mortem astrocytes: the involvement of prostaglandin E2," Brain Research, vol. 777, no. 1-2, pp. 210-218, 1997.

[63] B. L. Fiebich, M. Hüll, K. Lieb, K. Gyufko, M. Berger, and J. Bauer, "Prostaglandin E2 induces interleukin- 6 synthesis in human astrocytoma cells," Journal of Neurochemistry, vol. 68, no. 2, pp. 704-709, 1997.

[64] K. A. Kelley, L. Ho, D. Winger, et al., "Potentiation of excitotoxicity in transgenic mice overexpressing neuronal cyclooxygenase-2," American Journal of Pathology, vol. 155, no. 3, pp. 995-1004, 1999.

[65] G. M. Pasinetti, "Cyclooxygenase and inflammation in Alzheimer's disease: experimental approaches and clinical interventions," Journal of Neuroscience Research, vol. 54, no. 1, pp. 1-6, 1998.

[66] C. Jiang, A. T. Ting, and B. Seed, "PPAR- $\gamma$ agonists inhibit production of monocyte inflammatory cytokines," Nature, vol. 391, no. 6662, pp. 82-86, 1998.

[67] J. M. Lehmann, J. M. Lenhard, B. B. Oliver, G. M. Ringold, and S. A. Kliewer, "Peroxisome proliferator-activated receptors $\alpha$ and $\gamma$ are activated by indomethacin and other non-steroidal anti-inflammatory drugs," Journal of Biological Chemistry, vol. 272, no. 6, pp. 3406-3410, 1997.

[68] M. Ricote, A. C. Li, T. M. Willson, C. J. Kelly, and C. K. Glass, "The peroxisome proliferator-activated receptor- $\gamma$ is a negative regulator of macrophage activation," Nature, vol. 391, no. 6662, pp. 79-82, 1998.

[69] C. K. Combs, D. E. Johnson, S. B. Cannady, T. M. Lehman, and G. E. Landreth, "Identification of microglial signal transduction pathways mediating a neurotoxic response to amyloidogenic fragments of $\beta$-amyloid and prion proteins," Journal of Neuroscience, vol. 19, no. 3, pp. 928-939, 1999.

[70] C. K. Combs, D. E. Johnson, J. C. Karlo, S. B. Cannady, and G. E. Landreth, "Inflammatory mechanisms in Alzheimer's disease: inhibition of $\beta$-amyloid-stimulated proinflammatory responses and neurotoxicity by PPAR $\gamma$ agonists," Journal of Neuroscience, vol. 20, no. 2, pp. 558-567, 2000.

[71] M. A. Ansari and S. W. Scheff, "Oxidative stress in the progression of alzheimer disease in the frontal cortex," Journal of Neuropathology and Experimental Neurology, vol. 69, no. 2, pp. 155-167, 2010.

[72] M. A. Smith, X. Zhu, M. Tabaton, et al., "Increased iron and free radical generation in preclinical Alzheimer disease and mild cognitive impairment," Journal of Alzheimer's Disease, vol. 19, no. 1, pp. 353-372, 2010.

[73] H. M. Schipper, W. Song, H. Zukor, J. R. Hascalovici, and D. Zeligman, "Heme oxygenase-1 and neurodegeneration: expanding frontiers of engagement," Journal of Neurochemistry, vol. 110, no. 2, pp. 469-485, 2009.

[74] M. J. Alcaraz, P. Fernandez, and M. I. Guillén, "Antiinflammatory actions of the heme oxygenase-1 pathway," Current Pharmaceutical Design, vol. 9, no. 30, pp. 2541-2551, 2003.

[75] A. Cuadrado and A. I. Rojo, "Heme oxygenase-1 as a therapeutic target in neurodegenerative diseases and brain infections," Current Pharmaceutical Design, vol. 14, no. 5, pp. 429-442, 2008.
[76] K. Kimura, "Mechanisms of active oxygen species reduction by non-steroidal anti-inflammatory drugs," International Journal of Biochemistry and Cell Biology, vol. 29, no. 3, pp. 437-446, 1997.

[77] M. Nivsarkar, A. Banerjee, and H. Padh, "Cyclooxygenase inhibitors: a novel direction for Alzheimer's management," Pharmacological Reports, vol. 60, no. 5, pp. 692-698, 2008.

[78] C. Guardia-Laguarta, M. Pera, and Lleó A, " $\gamma$-secretase as a therapeutic target in Alzheimer's disease," Current Drug Targets, vol. 11, no. 4, pp. 506-517, 2010.

[79] S. Weggen, J. L. Eriksen, P. Das, et al., "A subset of NSAIDs lower amyloidogenic A $\beta 42$ independently of cyclooxygenase activity," Nature, vol. 414, no. 6860, pp. 212-216, 2001.

[80] T. Kukar, M. P. Murphy, J. L. Eriksen, et al., "Diverse compounds mimic Alzheimer disease-causing mutations by augmenting A $\beta 42$ production," Nature Medicine, vol. 11, no. 5, pp. 545-550, 2005.

[81] A. Lleó, O. Berezovska, L. Herl, et al., "Nonsteroidal antiinflammatory drugs lower $\mathrm{A} \beta 42$ and change presenilin 1 conformation," Nature Medicine, vol. 10, no. 10, pp. 10651066, 2004.

[82] P. L. McGeer, M. Schulzer, and E. G. McGeer, "Arthritis and anti-inflammatory agents as possible protective factors for Alzheimer's disease: a review of 17 epidemiologic studies," Neurology, vol. 47, no. 2, pp. 425-432, 1996.

[83] S. C. Vlad, D. R. Miller, N. W. Kowall, and D. T. Felson, "Protective effects of NSAIDs on the development of Alzheimer disease," Neurology, vol. 70, no. 19, pp. 1672-1677, 2008.

[84] B. K. Martin, C. Szekely, J. Brandt, et al., "Cognitive function over time in the Alzheimer's disease anti-inflammatory prevention trial (ADAPT): results of a randomized, controlled trial of naproxen and celecoxib," Archives of Neurology, vol. 65, no. 7, pp. 896-905, 2008.

[85] C. Wolfson, A. Perrault, Y. Moride, J. M. Esdaile, L. Abenhaim, and F. Momoli, "A case-control analysis of nonsteroidal anti-inflammatory drugs and Alzheimer's disease: are they protective?" Neuroepidemiology, vol. 21, no. 2, pp. 81-86, 2002.

[86] J. M. Craft, D. M. Watterson, and L. J. Van Eldik, "Human amyloid $\beta$-induced neuroinflammation is an early event in neurodegeneration," GLIA, vol. 53, no. 5, pp. 484-490, 2006.

[87] A. V. Yermakova and M. Kerry O’Banion, "Downregulation of neuronal cyclooxygenase-2 expression in end stage Alzheimer's disease," Neurobiology of Aging, vol. 22, no. 6, pp. 823-836, 2001.

[88] M. Combrinck, J. Williams, M. A. De Berardinis, et al., "Levels of CSF prostaglandin E2, cognitive decline, and survival in Alzheimer's disease," Journal of Neurology, Neurosurgery and Psychiatry, vol. 77, no. 1, pp. 85-88, 2006.

[89] P. S. Aisen, "The potential of anti-inflammatory drugs for the treatment of Alzheimer's disease," Lancet Neurology, vol. 1, no. 5, pp. 279-284, 2002. 


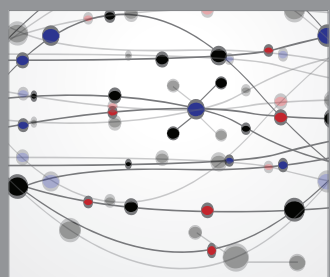

The Scientific World Journal
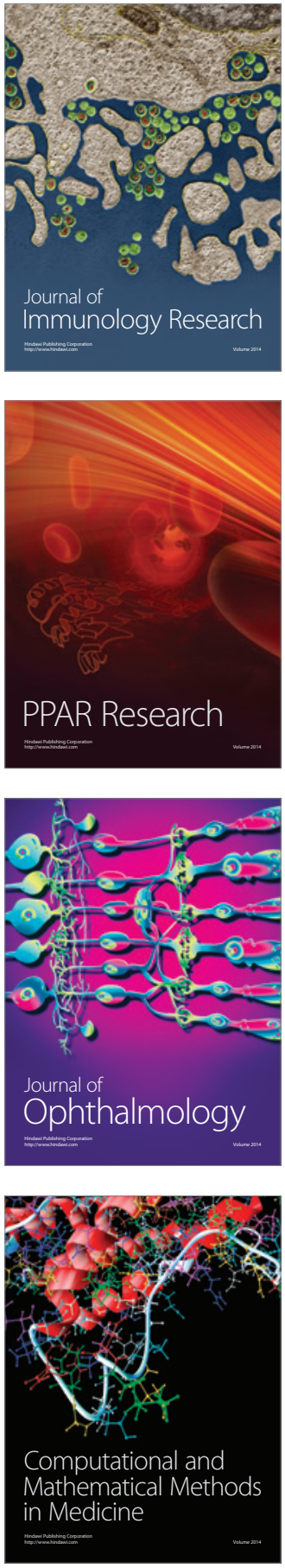

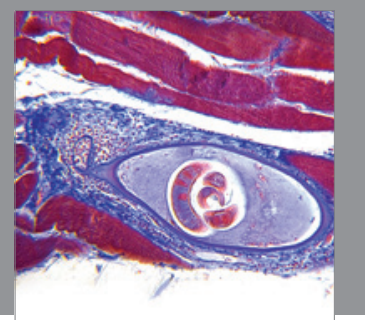

Gastroenterology

Research and Practice
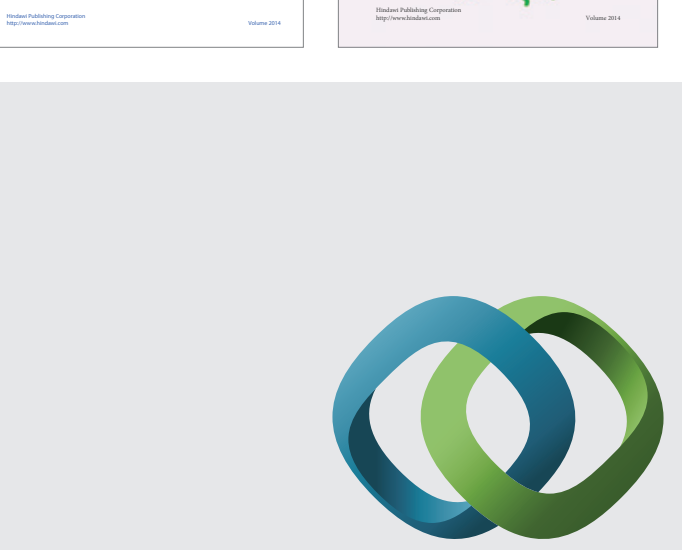

\section{Hindawi}

Submit your manuscripts at

http://www.hindawi.com
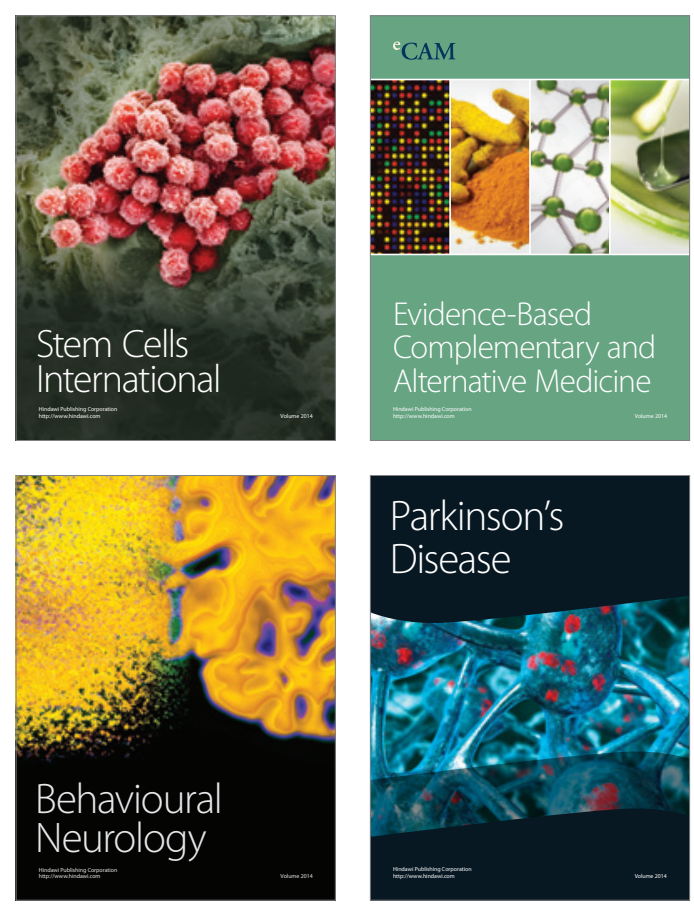

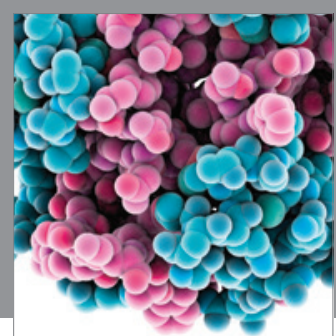

Journal of
Diabetes Research

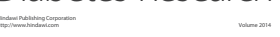

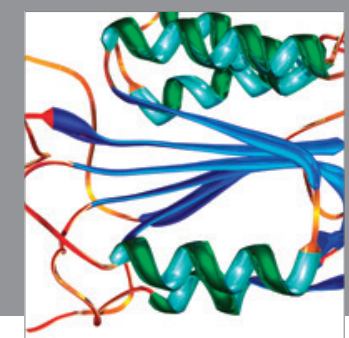

Disease Markers
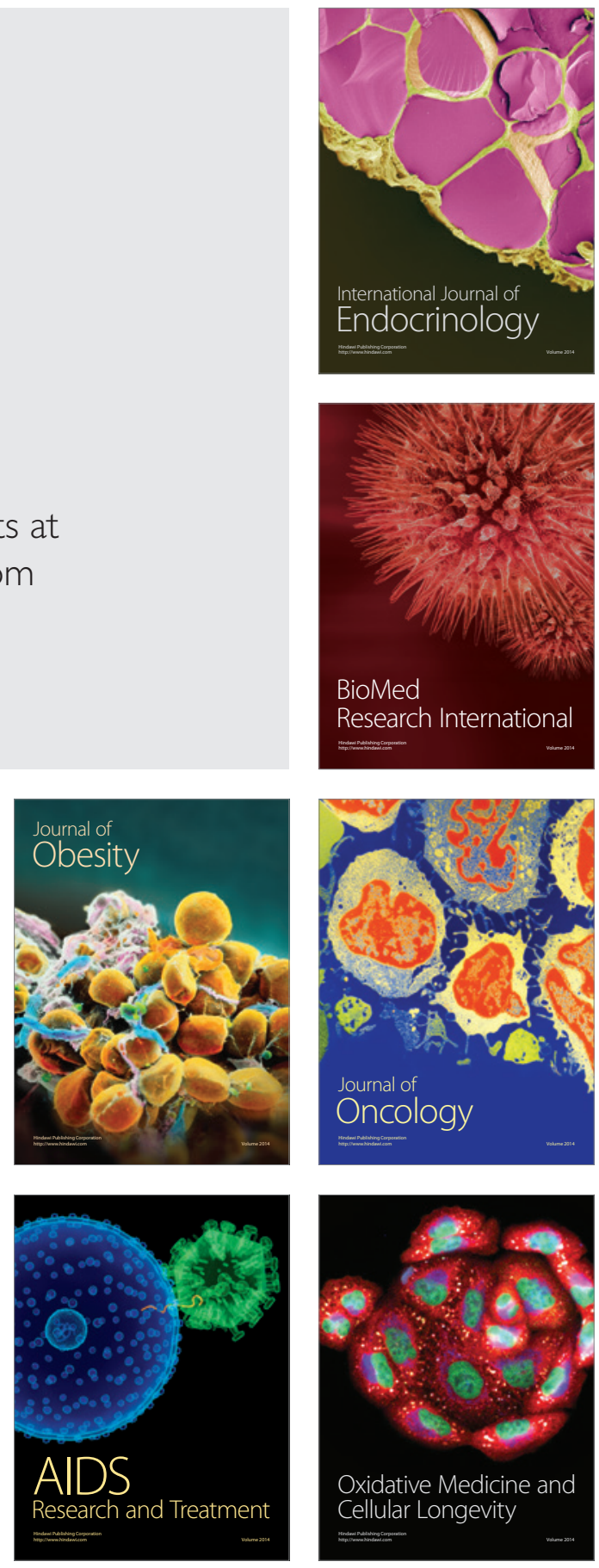